\title{
Two children with tremor
}

\author{
Helena Liu BSc, Tamara Pringsheim MD, Graham C. Thompson MD
}

\section{Competing interests: None \\ Case 1}

declared.

This article has been peer reviewed.

The authors have obtained patient consent.

Correspondence to: Graham Thompson, graham.thompson @ albertahealthservices.ca

CMAJ 2015. DOI:10.1503 /cmaj.120496
A right-handed 14-year-old boy was referred to a neurologist for an outpatient evaluation of tremor. The boy was previously healthy and not taking any medications. Developmental milestones were achieved within the appropriate norms. There was a family history of tremor in the boy's mother and maternal uncle. The mother reported that her tremor lessened following the consumption of alcohol. The parents first noticed the tremor in their son when he was about six years old. The tremor occurred mainly during voluntary activity, such as writing, eating soup with a spoon, drinking from a cup and using his hands for small skilled movements. The tremor affected both arms and was worse on the right side. His head, voice and legs were unaffected. The tremor was worse when he was physically tired (e.g., after heavy lifting) or nervous (e.g., public speaking). Over the years, the tremor has worsened slightly and has become a source of embarrassment to him.

On examination, the patient showed no distress and had normal vital signs; he was alert, behaved appropriately and had age-appropriate speech and language. Results of a cranial nerve examination were normal. A motor system examination showed normal bulk and tone. Strength in the upper and lower limbs was graded as 5 out of 5 in a manual muscle test. Results of sensory and gait examinations were normal. He was able to walk without difficulty using a tandem gait.

\section{KEY POINTS}

- Tremor is an uncommon presentation in children, accounting for $10 \%$ $20 \%$ of pediatric movement disorders.

- Although a broad range of diagnoses should be considered, essential, physiologic and post-traumatic tremors are the most common types of tremor seen in children.

- Thorough history and physical examination are essential to diagnose tremor; basic laboratory tests may help to rule out secondary causes when the cause is uncertain.

- Computed tomography or, preferably, magnetic resonance imaging should be ordered if a structural cause of tremor is suspected, which is often accompanied by additional signs of cerebellar or motor abnormalities.
There was no resting tremor. There was a highfrequency, low-amplitude postural tremor when his arms were outstretched to the front or sides and the tremor was slightly worse on the right side. Examination of the patient drinking from a paper cup showed tremor when he brought the cup to his mouth, with mild spillage of water. A writing examination showed mild tremor when the patient wrote cursively and when he drew a straight line and an Archimedean spiral.

Essential tremor was diagnosed. Basic laboratory tests to rule out secondary causes were performed, including measurement of the thyroid-stimulating hormone level to rule out hyperthyroidism, liver enzyme levels and the international normalized ratio (INR) to rule out hepatolenticular degeneration (which may occur in individuals with severe liver disease of any cause), and copper levels in a 24-hour urine collection to rule out Wilson disease (a specific entity that may include hepatolenticular degeneration). After discussion with his family, the patient elected to undergo treatment with a trial of propranolol (20 mg orally twice daily). At a follow-up examination two months later, the patient reported a substantial reduction $(75 \%)$ in tremor amplitude since starting treatment with propranolol. No hypotension or bradycardia was found on examination.

\section{Case 2}

A right-handed four-year-old girl presented to the emergency department with a four-week history of progressive intention tremor on the right side. Nine days earlier, she had been seen in the emergency department and dysmetria (i.e., a lack of accuracy with voluntary movements) was diagnosed (cause unknown). An urgent referral to outpatient neurology was arranged. At that time, an examination revealed intention tremor of her hands and right leg, as well as substantial pastpointing on finger-to-nose testing and an inability to trace the top of the leg on heel-to-shin testing. The tremor was more severe in her right hand. Her return visit was prompted by progressive symptoms, headache and a new onset of ataxia. 
Increased tiredness, difficulty finding words and blurry vision were also reported. Aside from having transient neonatal myasthenia gravis, she had been well previously. Perinatal, developmental and family histories were unremarkable.

On examination, she was not in distress, had normal vital signs and was alert and behaved appropriately. The results of a cranial nerve examination were normal. Funduscopic examination was normal, with no evidence of papilledema. A neurologic examination showed that the patient had difficulties with fine motor skills, a wide-based gait, an unsteady tandem gait, poor balance when standing on one foot and right footdrop. An intention tremor of the right hand was easily seen. The remainder of her examination was noncontributory.

Because of the progressive nature of the tremor and new onset of ataxia, an urgent computed tomography (CT) scan of the head was performed. Large, well-defined, isodense, bilateral thalamic masses without contrast enhancement were present, which suggested diffuse lowgrade glioma. Substantial mass effect was present with effacement of the third ventricle, which resulted in obstructive hydrocephalus. The patient was admitted to hospital for additional evaluation. Magnetic resonance imaging (MRI) showed bilateral, symmetric thalamic masses that were uniformly hypointense on $T_{1}$-weighted sequences and hyperintense on $T_{2}$-weighted and fluid-attenuated inversion recovery sequences, with no contrast enhancement after gadolinium administration (Figures 1 and 2). There were no indications for urgent surgical treatment of the hydrocephalus.

Neurosurgical resection was not feasible, and the risk involved with biopsy outweighed the need for tissue diagnosis, because the imaging was entirely consistent with low-grade glioma. Subsequently, the patient was followed by neuro-oncology for medical care, and treatment with topiramate was started. During her sevenday hospital stay, there was no history of vomiting or changes in level of consciousness. At discharge, the patient was prescribed topiramate, ranitidine and a tapering course of dexamethasone. Subsequently, she was referred to pediatric palliative care. The patient underwent a progressive neurologic deterioration and died 6 months after diagnosis.

\section{Discussion}

Tremor is one of the most common movement disorders seen in clinical practice, but it is an uncommon reason to seek medical attention during childhood, accounting for just $10 \%-20 \%$ of pediatric movement disorders. ${ }^{1}$ In a crosssectional study involving 819 school-aged children in Spain, the prevalence of tremor was evaluated through the assessment of spiral drawings; mild to moderate tremor was observed in $2.1 \%$ of the children. ${ }^{2}$ Tremor is defined as a rhythmic and sinusoidal movement produced by reciprocally innervated antagonist muscles. The rhythmicity of tremor is the most recognizable feature and distinguishes it from other pediatric movement disorders. ${ }^{1}$ Childhood tremor can occur with a wide variety of pathologic processes. The approach to diagnosing tremor in children similar to that in adults; $;, 4$ however, there are important differences in the causes of tremor in the pediatric population.

\section{Tremor classification}

Tremors are classified as rest or action tremors depending on the circumstances under which they occur. ${ }^{3}$ Rest tremor occurs when a limb is relaxed and supported against gravity (e.g., a hand rested on a table). It is often augmented by mental stress and attenuates with action. Most tremors are action tremors, which occur with voluntary muscle contractions. They can be further subdivided into postural, isometric, kinetic or task-specific tremors. A postural tremor is observed while maintaining a position against

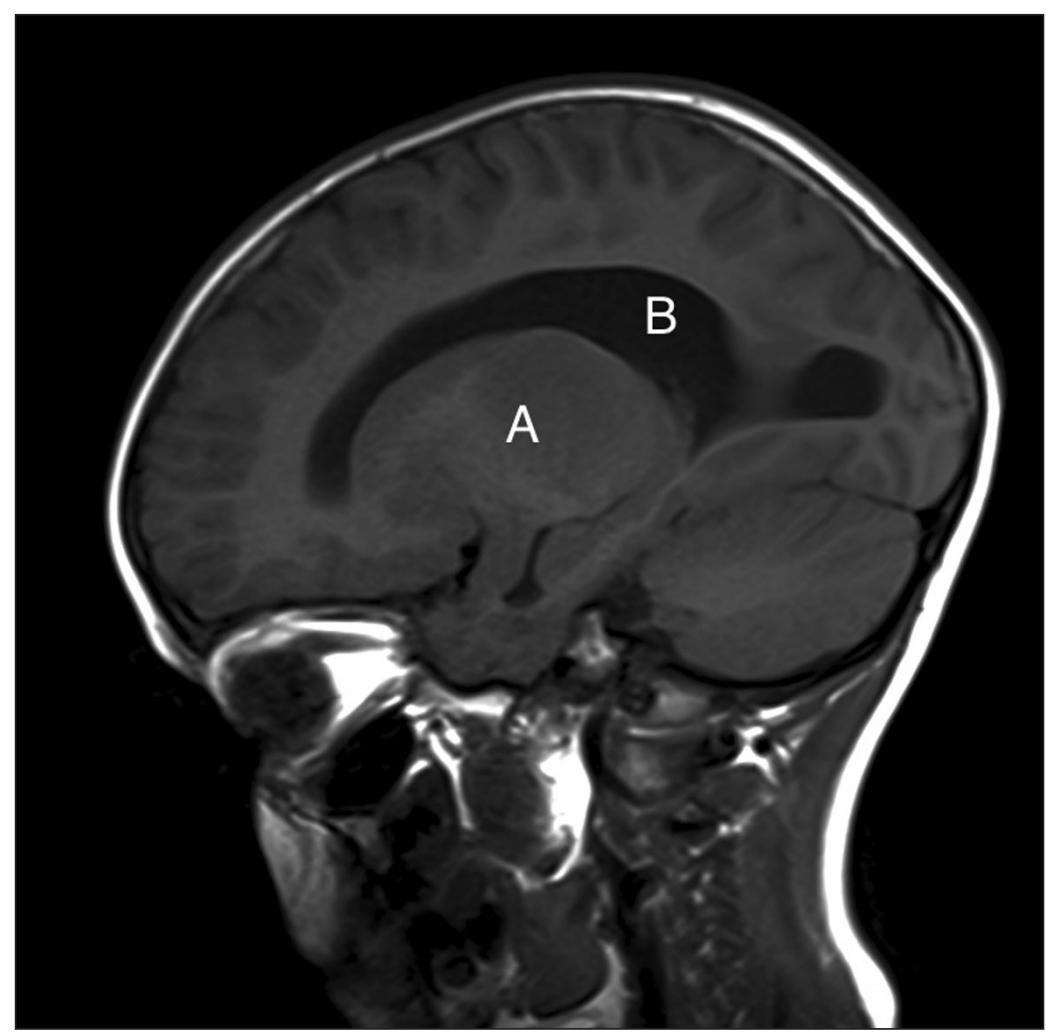

Figure 1: Magnetic resonance imaging of the head (sagittal view) in a fouryear-old girl with intention tremor. Note the large, bilateral thalamic masses (A) and resultant hydrocephalus (B). 
gravity. An isometric tremor occurs when muscles contract against rigid stationary objects. A kinetic tremor occurs during voluntary muscle contraction; kinetic tremor is considered "simple" when it occurs during non-target-directed movements (e.g., flexion or extension at wrist) and "intentional" when it is target-directed (e.g., finger-to-nose test). Task-specific tremor occurs only during highly skilled activities (Box 1)., ${ }^{1,5}$

A video showing tremor in a 15 -year-old boy can be found in Appendix 1 (available at www .cmaj.ca/lookup/suppl/doi:10.1503/cmaj.120496 /-/DC1). The video shows postural and intention tremor, ataxia and tremor occuring when the patient writes with his right hand. An image of the Archimedean spiral drawn by this patient can be found in Appendix 2 (available at www.cmaj.ca /lookup/suppl/doi:10.1503/cmaj.120496/-/DC1.

\section{Common childhood tremors}

A wide range of conditions can cause tremor. The most common cause of action tremors in the general population is essential tremor, affecting $0.4 \%-4.0 \%$ of the population. ${ }^{6}$ It is also the most common tremor in children, followed by physio-

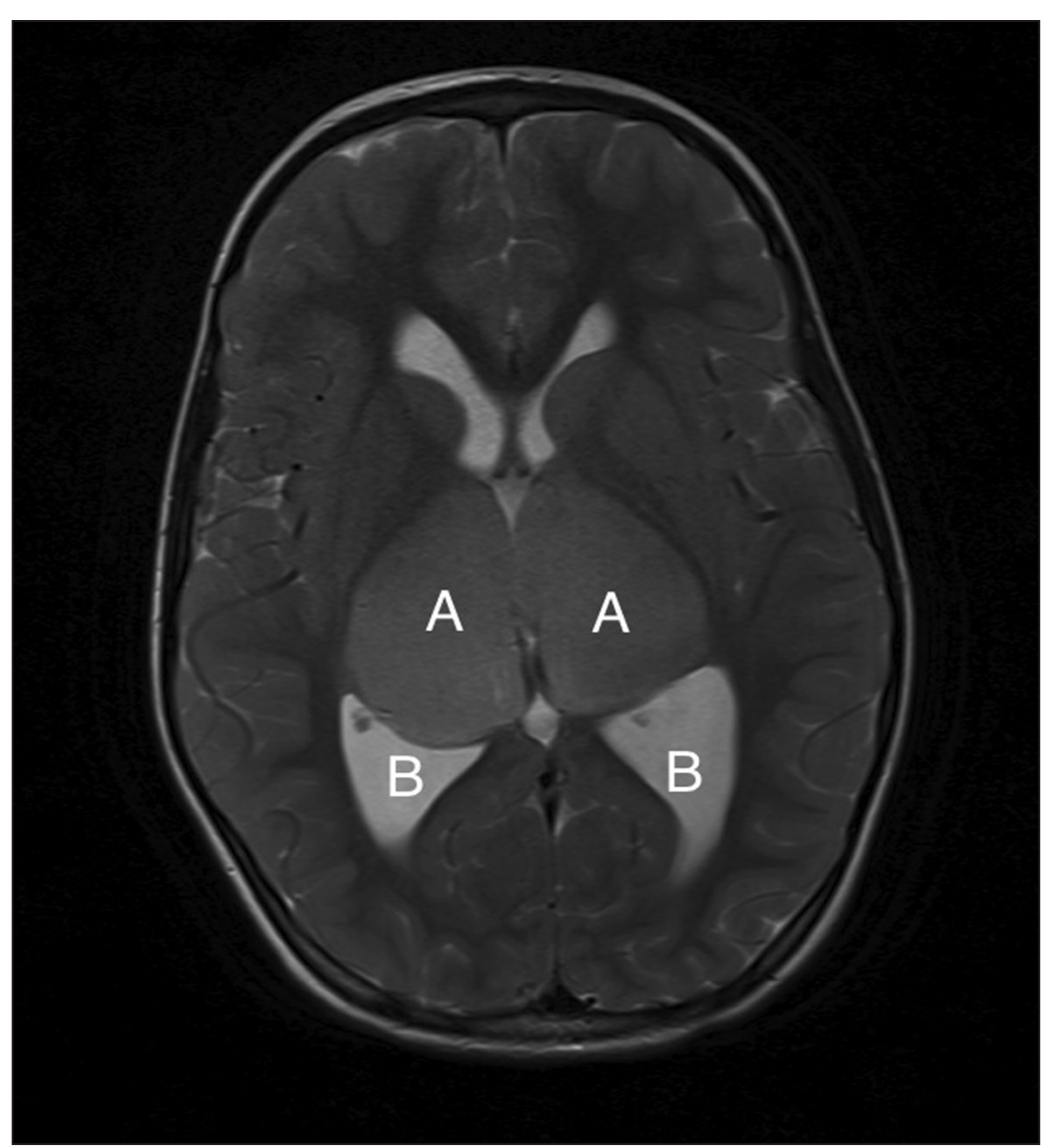

Figure 2: Magnetic resonance imaging with gadolinium of the head (transverse view) in a four-year-old girl with intention tremor. Note the large, bilateral thalamic masses (A) and resultant hydrocephalus (B). logic, primary writing and post-traumatic tremors. ${ }^{6}$ Less common causes of tremor in children include juvenile Parkinson disease and Wilson disease. Intention tremor due to malignant brain tumours is rare.

\section{Essential tremor}

Essential tremor develops insidiously and progresses slowly, presenting as a postural tremor involving both hands and arms in $95 \%$ of patients, although asymmetry may be present. It may appear as early as two years of age but often starts in late childhood or adolescence. Children with essential tremor often have a family history of the same. ${ }^{6}$ The common clinical presentation is the gradual onset of postural tremor over several years that involves both hands and arms and is occasionally associated with tremor of the head or voice. Leg tremor rarely occurs in essential tremor, but it can be a presenting feature of juvenile Parkinson disease. Gait is normal in the early and middle stages of essential tremor, but difficulties with tandem gait can arise in later adulthood. ${ }^{5}$ The amplitude of essential tremor typically increases with stress, fatigue and medications that stimulate the central nervous system, and with certain voluntary activities, such as holding a cup. Resting or drinking alcohol decreases amplitude. Studies have shown that individuals with essential tremor lack structural or cellular abnormalities but may display cerebellar and cerebellar-pathway dysfunction. One hypothesis is that the condition is due to the central oscillator being enhanced or suppressed by reflex pathways. ${ }^{6,7}$ According to the American Academy of Neurology guidelines, treatment with $\beta$-blockers (e.g., propranolol) or anticonvulsants (e.g., primadone) should be started when the tremor results in functional disability or social embarrassment (Appendix 3 available at www.cmaj.ca/lookup/suppl/doi:10.1503/cmaj $.120496 /-/ D C 1){ }^{8}{ }^{89}$ Although these guidelines are based on studies involving adults, a paucity of studies involving children necessitates that current practice extrapolate this evidence to tremor in children.

\section{Physiologic tremor}

All individuals exhibit physiologic tremor, a benign, low-amplitude, high-frequency postural tremor that is barely visible. Physiologic tremor is thought to arise from the resonant oscillation of a limb because of mechanical factors affecting it, such as firing of the motor units of muscles and cardioballistic effects. ${ }^{10}$ It can be enhanced to the point of detection with stress and anxiety, such as before a classroom presentation, or with central nervous system stimulants and adrenergic 
agents. Removal of the underlying causes and reassurance from family members are the basis of managing physiologic tremor.

\section{Post-traumatic tremor}

Different types of tremor occur after an injury to the head, with the most debilitating type having a low frequency and a high amplitude, which often interferes with ordinary activities. It is thought that damage to dentatorubrothalamic pathways in the midbrain is the basis of post-traumatic intention tremor. ${ }^{11}$ Important predictive factors for the development of tremor after head injury include severity of trauma, loss of consciousness, coma and residual neurologic deficits. ${ }^{6}$ Initial treatment should include medications such as levodopa or clonazepam, and if necessary, surgical options may be considered (Appendix 3). ${ }^{6,8}$

\section{Rare childhood tremors}

\section{Intention tremor}

Intention tremor commonly presents in both arms and legs and may also affect the head. It is typically low frequency $(3-5 \mathrm{~Hz})$, although a mild postural tremor that is more rapid $(10 \mathrm{~Hz})$ may also be present. ${ }^{12}$ Intention tremor is typically caused by cerebellar lesions (e.g., tumours, trauma, multiple sclerosis and stroke) or intoxication caused by the use of drugs or alcohol (Box 1). ${ }^{5}$ Inherited disorders resulting in spinocerebellar degeneration, such as Friedreich ataxia, or spinocerebellar ataxias can also produce intention tremor, although the onset of symptoms is gradual and occurs in combination with other neurologic deficits.

In an examination of patients with intention tremor, associated symptoms such as dysarthria, nystagmus, imbalance, dysmetria, ataxia and broad-based gait ${ }^{13}$ (Box 2) should also be investigated, because these additional signs and symptoms may help point to cerebellar abnormalities. ${ }^{14}$ Evaluation for additional motor signs, such as rigidity or bradykinesia, may suggest a disorder that affects both the cerebellum and the extrapyramidal system. Intention tremor is best elicited during finger-to-nose or heel-to-shin tests. For younger children with minimum cooperation, having them drink from a full cup or eat cereal with a spoon can also be illustrative. ${ }^{1}$ Once intention tremor is noted, CT or MRI is the most important tool for ruling out focal lesions (Appendix 3). ${ }^{8}$ In situations where treating the underlying disease is feasible, tremor may improve after the pathologic cause is removed.

Our second case illustrates a rare presenta-

Box 1: Classification of tremor in children ${ }^{5,6,12}$

\begin{tabular}{|c|c|c|c|}
\hline Type of tremor & Frequency $(\mathrm{Hz})$ & Occurrence & Examples \\
\hline Rest & Low-medium (3-6) & $\begin{array}{l}\text { While limb is relaxed and } \\
\text { supported against gravity }\end{array}$ & $\begin{array}{l}\text { - Juvenile Parkinson disease } \\
\text { - Medication induced (e.g., use of lithium, neuroleptics, } \\
\text { anticonvulsants, immunosuppressants or } \beta \text {-adrenergic } \\
\text { antagonists) } \\
\text { - Wilson disease }\end{array}$ \\
\hline Action & & $\begin{array}{l}\text { During voluntary muscle } \\
\text { contraction }\end{array}$ & \\
\hline Postural & Medium-high (4-12) & $\begin{array}{l}\text { While limb is held in } \\
\text { position against gravity }\end{array}$ & $\begin{array}{l}\text { - Physiologic tremor } \\
\text { - Essential tremor } \\
\text { - Drug withdrawal } \\
\text { - Neuropathy } \\
\text { - Metabolic disturbance }\end{array}$ \\
\hline \multicolumn{4}{|l|}{ Kinetic } \\
\hline Isometric & Medium (4-6) & $\begin{array}{l}\text { During muscle contraction } \\
\text { against stationary objects }\end{array}$ & $\begin{array}{l}\text { - Essential tremor } \\
\text { - Enhanced physiologic tremor } \\
\text { - Juvenile Parkinson disease }\end{array}$ \\
\hline Task specific & Variable (4-10) & $\begin{array}{l}\text { During task-specific limb } \\
\text { movements }\end{array}$ & $\begin{array}{l}\text { - Handwriting tremor } \\
\text { - Musician's tremor }\end{array}$ \\
\hline
\end{tabular}


tion of intention tremor secondary to bilateral thalamic gliomas in a young girl. Bilateral thalamic gliomas are large, low-grade, slowgrowing tumours located in symmetric areas of the bilateral thalami. One literature review suggested that these tumours typically manifest in children as movement disorders, whereas adults show behavioural impairments varying from personality changes to dementia. ${ }^{15}$ Tremors occurring secondary to these gliomas can be explained by infiltration of the tumours into ventroanterior and centrolateral thalamic nuclei and dentatorubrothalamic tract. ${ }^{15}$

\section{Diagnostic approach}

Following an initial examination to ensure the patient is medically stable, a thorough history should be elicited of the onset of tremor, including precipitating, aggravating and relieving factors such as trauma, alcohol intake, medications, stress and fatigue. Family history, associated symptoms and functional limitations, such as social embarrassment or difficulty with holding a cup of water, should also be explored. A physical examination should be done to evaluate the location (arms, legs, head and neck), frequency (fast or slow), regularity (rhythmic or nonrhythmic) and amplitude (fine or coarse) of the tremor. Tremor in the arms should be observed with the affected limb fully supported at rest, with the limb elevated against gravity and while the patient performs goal-directed movements. Tremor in the legs should be evaluated with the legs at rest, during heel-to-shin testing and while the patient is standing and walking. ${ }^{5,8}$

Postural and other action tremors are best elicited with the patient's arms outstretched, during finger-to-nose manoeuvres, and while the patient drinks from a cup and performs written tasks. With intention tremor, associated symptoms such as nystagmus, dysarthria, dysmetria and gait ataxia should be investigated. When examining patients with resting tremor, it is important to check for associated rigidity and bradykinesia, which may suggest juvenile Parkinson disease or Wilson disease. For younger children, when some of these assessments may not be feasible because of a lack of cooperation or failure to have achieved the necessary motor skills, it is helpful to ask the family or caregivers whether these behaviours have been observed previously. Activities such as building towers with cubes, tracing a simple figure with crayons or even tracing a path through a maze will provide clear evidence of the presence of a tremor. In the situation where the tremor in the child is not adequately observed, asking the parents or caregivers to record video evidence of the child at home performing activities that best elicit the tremors is valuable in the assessment process. ${ }^{1}$

The diagnosis of tremor is clinical but can be supported by laboratory tests and diagnostic imaging. Standard laboratory evaluation of tremor should include a chemistry profile (i.e., levels of glucose and liver enzymes and INR), complete blood count and thyroid function tests. ${ }^{5}$ Addi-

\begin{tabular}{|c|c|c|}
\hline Tremor syndrome & Clinical features & Diagnostic approach \\
\hline Physiologic tremor & Postural tremor: no neurologic defects & $\begin{array}{l}\text { - Chemistry profile } \\
\text { - Thyroid function test } \\
\text { - Review of current medications }\end{array}$ \\
\hline Essential tremor & $\begin{array}{l}\text { Postural tremor: affects both hands } \\
\text { and arms and sometimes the head; } \\
\text { increases in amplitude with stress, } \\
\text { fatigue and stimulants, and decreases } \\
\text { with alcohol }\end{array}$ & $\begin{array}{l}\text { - No specific tests } \\
\text { - Rule out other causes }\end{array}$ \\
\hline Juvenile Parkinson disease & $\begin{array}{l}\text { Resting tremor: increases in amplitude } \\
\text { with stress and disappears with } \\
\text { voluntary movement of limb }\end{array}$ & $\begin{array}{l}\text { - No specific testing needed for typical presentation } \\
\text { - MRI for atypical presentations } \\
\text { - Rule out other causes }\end{array}$ \\
\hline Cerebellar tremor & $\begin{array}{l}\text { Intention tremor: affects both arms } \\
\text { and legs and sometimes the head; } \\
\text { results of finger-to-nose and heel-to- } \\
\text { shin testing are abnormal; gait } \\
\text { abnormalities; dysarthria; nystagmus }\end{array}$ & $\begin{array}{l}\text { - CT or MRI } \\
\text { - Examination of cerebrospinal fluid for IgG gamma } \\
\text { globulins (if multiple sclerosis is suspected) } \\
\text { - Levels of toxins and alcohol (if intoxication suspected) }\end{array}$ \\
\hline Wilson disease & $\begin{array}{l}\text { Wing-beating tremor: associated with } \\
\text { signs of hepatic disease (e.g., ascites or } \\
\text { jaundice) }\end{array}$ & $\begin{array}{l}\text { - Liver function test } \\
\text { - Serum ceruloplasmin level } \\
\text { - Measurement of copper levels in urine } \\
\text { - Slit-lamp examination }\end{array}$ \\
\hline
\end{tabular}


tional laboratory tests for patients with suspected Wilson disease should include liver enzymes and function tests, copper level in a 24-hour collection of urine and the serum level of ceruloplasmin. In patients with suspected multiple sclerosis, examination of the cerebrospinal fluid for oligoclonal IgG bands is appropriate. Screening for toxins and alcohol also may be warranted (Appendix 3) ${ }^{5,8}$ Imaging of the brain should be ordered if a structural or metabolic cause of tremor is suspected, such as space-occupying lesions, multiple sclerosis, trauma or stroke. Urgent CT is often used in acute care settings, but it should be used judiciously because of the risk of substantial radiation exposure. ${ }^{16}$ The preferred modality is MRI if time allows, keeping in mind that small children will require general anesthesia for this test.

Referral to a neurologist should occur if the cause of the tremor is uncertain, the cause requires long-term neurology support (i.e., multiple sclerosis or stroke) or if the tremor interrupts the child's daily life indicating the need for treatment. Neurosurgical evaluation should be started promptly for children with spaceoccupying lesions.

\section{Conclusion}

Tremor in children has many of the same clinical features as in adults, although it is an uncommon presentation. Early recognition of childhood tremor and the associated signs and symptoms is essential to accurately identify the underlying cause and formulate an effective treatment plan.

\section{References}

1. Keller S, Dure LS. Tremor in childhood. Semin Pediatr Neurol 2009; 16:60-70.

2. Louis ED, Cubo E, Trejo-Gabriel-Galan JM, et al. Tremor in school-aged children: a cross-sectional study of tremor in 819 boys and girls in Burgos, Spain. Neuroepidemiology 2011; 37:90-5.

3. Kumar H, Jog M. A patient with tremor, part 1: making the diagnosis. CMAJ 2011 183:1507-10.

4. Kumar H, Jog M. A patient with tremor, part 2: from diagnosis to treatment. CMAJ 2011;183:1612-16.

5. Sirisena D, Williams DR. My hands shake - classification and treatment of tremor. Aust Fam Physician 2009;38:678-83.

6. Uddin MK, Rodnitzky RL. Tremor in children. Semin Pediatr Neurol 2003;10:26-34.

7. Louis ED, Bonsattel JPG. The emerging neuropathology of essential tremor. Mov Disord 2008;23:174-82.

8. Anouti A, Koller WC. Diagnostic testing in movement disorders. Neurol Clin 1996;14:169-82.

9. Zesiewicz TA, Elble RJ, Louis ED, et al. Evidence-based guideline update: treatment of essential tremor: report of the Quality Stan- dards Subcommittee of the American Academy of Neurology. Neurology 2011;77:1752-5.

10. Raethjen J, Lindemann M, Schmaljohann H, et al. Multiple oscillators are causing parkinsonian and essential tremor. Mov Disord 2000;15:84-94.

11. Iwadate Y, Saeki N, Namba H, et al. Post-traumatic intention tremor - clinical features and CT findings. Neurosurg Rev 1989;12(Suppl 1):500-7.

12. Bhidayasiri R. Differential diagnosis of common tremor syndromes. Postgrad Med J 2005;81:756-62.

13. Venes D, editor. Taber's cyclopedic medical dictionary. 21st ed. Philadelphia: F.A. Davis Company; 2009:205, 703, 708, 918, 1613.

14. Weiss N, North RB, Ohara S, et al. Attenuation of cerebellar tremor with implantation of an intrathecal baclofen pump: the role of gamma-aminobutyric acidergic pathways. Case report. J Neurosurg 2003;99:768-71.

15. Menon G, Nair S, Krishnamoorthy T, et al. Bilateral thalamic glioma: report of four cases and review of literature. J Pediatr Neurosci 2006;1:66-9.

16. Brenner DJ, Hall EJ. Computed tomography - An increasing source of radiation exposure. N Engl J Med 2007;357:2277-84.

Affiliations: Faculty of Medicine and Dentistry (Liu), University of Alberta, Edmonton, Alta.; Departments of Clinical Neurosciences (Pringsheim) and Pediatrics (Pringsheim, Thompson), Faculty of Medicine, University of Calgary, Calgary, Alta; Pediatric Emergency Medicine (Thompson), Departments of Clinical Neurosciences and Pediatrics (Pringsheim), Alberta Children's Hospital, Calgary, Alta.

Contributors: Helena Liu performed the background literature review. Tamara Pringsheim contributed one of the case studies and critically reviewed the manuscript for intellectual content (neurology). Graham Thompson was the supervising author, conceived the manuscript and critically revised it for intellectual content (emergency medicine), and contributed one of the case studies. All of the authors participated in drafting and revising the manuscript, approved the final version submitted for publication and agreed to act as guarantors of the work.

Acknowledgements: The authors thank the families for their courage in sharing their children's stories.

A video showing examples of tremor in the right hand of a 15 -year-old boy with a history of a right cerebellar pilocytic astrocytoma is available in Appendix 1, at www.cmaj.ca/lookup/suppl/doi:10.1503 /cmaj.120496/-/DC1.

The section Cases presents brief case reports that convey clear, practical lessons. Preference is given to common presentations of important rare conditions, and important unusual presentations of common problems. Articles start with a case presentation (500 words maximum), and a discussion of the underlying condition follows (1000 words maximum). Visual elements (e.g., tables of the differential diagnosis, clinical features or diagnostic approach) are encouraged. Written consent from patients for publication of their story is a necessity and should accompany submissions. See information for authors at www.cmaj.ca. 